\title{
Strong-strong beam-beam simulation using a Green function approach
}

\author{
Ji Qiang, Miguel A. Furman, and Robert D. Ryne \\ Accelerator and Fusion Research Division, Lawrence Berkeley National Laboratory, Berkeley, California 94720
}

(Received 13 August 2002; published 28 October 2002)

\begin{abstract}
In this paper we present a new approach, based on a shifted Green function, to evaluate the electromagnetic field in a simulation of colliding beams. Unlike a conventional particle-mesh code, we use a method in which the computational mesh covers only the largest of the two colliding beams. This allows us to study long-range parasitic collisions accurately and efficiently. We have implemented this algorithm in a new parallel strong-strong beam-beam simulation code. As an application, we present a study of a beam sweeping scheme for the LBNL luminosity monitor of the Large Hadron Collider.
\end{abstract}

DOI: $10.1103 /$ PhysRevSTAB.5.104402

PACS numbers: 29.27.Bd

\section{INTRODUCTION}

The beam-beam interaction puts a strong limit on the performance of most colliders. At the interaction points (IPs), the electromagnetic fields generated by one beam focus or defocus the beam moving in the opposite direction. This can cause beam blowup and a reduction of luminosity. An accurate simulation of the beam-beam interaction will help to optimize the luminosity in high energy colliders. Macroparticle tracking provides an invaluable tool for the study of beam-beam interactions. In this approach, a number of simulation particles are generated with the same charge-to-mass ratio as the real particles. Outside the interaction region, each particle is transported using transfer maps associated with external elements, radiation damping, and quantum excitation. At the interaction point, the electromagnetic fields from the colliding beams are calculated and applied to the opposite beam. To calculate the electromagnetic field requires the solution of the Poisson equation after each turn. The soft Gaussian approximation is usually used to obtain the electromagnetic fields quickly [1-8]. To take into account the effects of the beam distribution self-consistently, one has to solve the Poisson equation numerically. Particlemesh methods have been used to solve the Poisson equation on a numerical grid [9-13]. Using these methods, the computational cost for the Poisson solver can be on the order of $N_{g}^{2} \log \left(N_{g}\right)$, where $N_{g}$ is the number of grid points in one direction. Since these methods normally require using a mesh covering both beams, the straightforward application of such an approach would have strong disadvantages for two beams with large separation as in the long-range interaction [14]. Given that the electromagnetic fields in the empty space between the two beams are not needed, such an approach would be a waste of computational power and would furthermore lead to poor numerical resolution on the grid for widely spaced beams.

$$
\left(\begin{array}{c}
x \\
x^{\prime}
\end{array}\right)_{n+1}=\left(\begin{array}{cc}
\cos \left(2 \pi \nu_{0 x}\right)+\alpha_{x} \sin \left(2 \pi \nu_{0 x}\right) & \beta_{x} \sin \left(2 \pi \nu_{0 x}\right) \\
-\gamma_{x} \sin \left(2 \pi \nu_{0 x}\right) & \cos \left(2 \pi \nu_{0 x}\right)-\alpha_{x} \sin \left(2 \pi \nu_{0 x}\right)
\end{array}\right)\left(\begin{array}{c}
x \\
x^{\prime}
\end{array}\right)_{n},
$$

where $\alpha_{x}, \beta_{x}$, and $\gamma_{x}$ are lattice functions at the interaction point, $\nu_{0 x}$ is the horizontal lattice tune, and $n$ is the turn
A hybrid fast multipole method has been proposed to calculate the electromagnetic field [14]. In this paper, we propose a particle-mesh method using a shifted Green function. Using such an approach, the computational domain covers only the size of the largest beam. This avoids the potential loss of numerical resolution and leads to high computational efficiency since the fields between the bunches are not calculated.

The organization of this paper is as follows: The physical model and computational method are described in Sec. II. Applications to the study of coherent beam-beam oscillations and beam sweeping in the Large Hadron Collider (LHC) are given in Sec. III. The conclusions are drawn in Sec. IV.

\section{PHYSICAL MODEL AND COMPUTATIONAL METHOD}

In our beam-beam simulation, each charged particle has a position in phase space with coordinates $\left(x, x^{\prime}, y, y^{\prime}, \Delta z / \sigma_{z}, \Delta p_{z} / \sigma_{p_{z}}\right)$. Here, a superscript prime denotes $\partial / \partial s$, where $s$ is the arc length. The motion of a particle will be subject to the influence of external fields, which provide transverse and longitudinal focusing of the beam. A particle will also lose energy through synchrotron radiation, i.e., through radiation damping and quantum excitation. The Coulomb interaction among charged particles within a bunch is negligible due to the cancellation of electric and magnetic forces at high energy. However, for the opposite-moving particles, the electric and magnetic forces will add up. This can significantly affect the motion of the particles in the other beam.

The effects of the external field can be represented, in the small-amplitude approximation, by a one-turn linear map. For our model, the map governing the horizontal motion is given by [3] 
number. A similar map applies to the vertical phase space $y$ and $y^{\prime}$ by replacing $x \rightarrow y$ in the above equation. For the longitudinal phase space, the one-turn map is defined by

$$
\left(\begin{array}{c}
\Delta z / \sigma_{z} \\
\Delta p_{z} / \sigma_{p_{z}}
\end{array}\right)_{n+1}=\left(\begin{array}{cc}
\cos \left(2 \pi \nu_{s}\right) & \sin \left(2 \pi \nu_{s}\right) \\
-\sin \left(2 \pi \nu_{s}\right) & \cos \left(2 \pi \nu_{s}\right)
\end{array}\right)\left(\begin{array}{c}
\Delta z / \sigma_{z} \\
\Delta p_{z} / \sigma_{p_{z}}
\end{array}\right)_{n}
$$

where $\nu_{s}$ is the synchrotron tune.

The effects of radiation damping and quantum excitation can be represented using a localized stochastic map. For $\alpha_{x}=\alpha_{y}=0$, the map consists of the following transformations [3]:

$$
\begin{gathered}
x_{n+1}=\lambda_{x} x_{n}+r_{1} \sigma_{x} \sqrt{1-\lambda_{x}^{2},} \\
x_{n+1}^{\prime}=\lambda_{x^{\prime}} x_{n}^{\prime}+r_{2} \sigma_{x^{\prime}} \sqrt{1-\lambda_{x^{\prime}}^{2},} \\
y_{n+1}=\lambda_{y} y_{n}+r_{3} \sigma_{y} \sqrt{1-\lambda_{y}^{2}}, \\
y_{n+1}^{\prime}=\lambda_{y^{\prime}} y_{n}^{\prime}+r_{4} \sigma_{y^{\prime}} \sqrt{1-\lambda_{y^{\prime}}^{2}} \\
\Delta z_{n+1}=\lambda_{z} \Delta z_{n}+r_{5} \sigma_{z} \sqrt{1-\lambda_{z}^{2}}, \\
\Delta p_{z_{n+1}}=\lambda_{p_{z}} \Delta p_{z_{n}}+r_{6} \sigma_{p_{z}} \sqrt{1-\lambda_{p_{z}}^{2}},
\end{gathered}
$$

where the $\sigma$ 's are the nominal rms equilibrium beam sizes in each dimension, the $\lambda$ 's are given in terms of the damping time $\tau$ (measured in units of turns) by $\lambda_{i}=$ $\exp \left(-1 / \tau_{i}\right)$ where $i$ denotes $x, y$, or $z$, and the $r$ 's are independent random numbers satisfying

$$
\begin{gathered}
\left\langle r_{i}\right\rangle=0, \\
\left\langle r_{i} r_{j}\right\rangle=\delta_{i j} .
\end{gathered}
$$

The first term in the above transformation represents the radiation damping, and the second term represents the quantum excitation.

After applying the previous maps, the slopes of the particles are updated,

$$
\begin{aligned}
& x_{\text {new }}^{\prime}=x^{\prime}+\Delta x^{\prime}, \\
& y_{\text {new }}^{\prime}=y^{\prime}+\Delta y^{\prime},
\end{aligned}
$$

due to the beam-beam electromagnetic forces at the collision point. Here, the kick on a particle in beam 1 due to the influence of beam 2 is given by

$$
\begin{aligned}
& \Delta x_{1}^{\prime}=\frac{2 q_{1} q_{2} N_{2}}{\gamma_{1} 4 \pi \epsilon_{0} m_{1} c^{2}} E_{x_{2}}, \\
& \Delta y_{1}^{\prime}=\frac{2 q_{1} q_{2} N_{2}}{\gamma_{1} 4 \pi \epsilon_{0} m_{1} c^{2}} E_{y_{2}},
\end{aligned}
$$

where $\gamma$ is the relativistic factor, $c$ is the speed of light, $\epsilon_{0}$ is the vacuum permittivity, $q$ is the charge of a particle, $m$ is the rest mass of a particle, $N$ is the number of particles in a bunch, and $E_{x}$ and $E_{y}$ are the transverse electric fields generated by the opposing beam. The electric fields can be obtained from the solution of the two-dimensional Poisson equation:

$$
\begin{gathered}
\frac{\partial^{2} \phi}{\partial x^{2}}+\frac{\partial^{2} \phi}{\partial y^{2}}=-2 \pi \rho(x, y), \\
\mathbf{E}=-\nabla \phi,
\end{gathered}
$$

where $\phi$ is the two-dimensional electric potential and $\rho$ is the transverse particle density. The corresponding expression for the influence of beam 1 on particles in beam 2 is obtained by exchanging the subscripts 1 and 2 .

The general solution of Poisson's equation can be written as

$$
\phi(x, y)=\int G(x, \bar{x}, y, \bar{y}) \rho(\bar{x}, \bar{y}) d \bar{x} d \bar{y},
$$

where $G$ is Green's function. For the case of transverse open boundary conditions, Green's function is given by

$$
G(x, \bar{x}, y, \bar{y})=-\frac{1}{2} \ln \left[(x-\bar{x})^{2}+(y-\bar{y})^{2}\right] .
$$

Now consider a simulation of an open system where the computational domain containing the particles has a range of $\left(0, L_{x}\right)$ and $\left(0, L_{y}\right)$, and where each dimension has been discretized using $N_{x}$ and $N_{y}$ points. From Eq. (17), the electric potentials on the grid can be approximated as

$$
\phi\left(x_{i}, y_{j}\right)=h_{x} h_{y} \sum_{i^{\prime}=1}^{N_{x}} \sum_{j^{\prime}=1}^{N_{y}} G\left(x_{i}-x_{i^{\prime}}, y_{i}-y_{j^{\prime}}\right) \rho\left(x_{i^{\prime}}, y_{j^{\prime}}\right),
$$

where $x_{i}=(i-1) h_{x}$ and $y_{j}=(j-1) h_{y}$. This convolution can be replaced by a cyclic convolution expression in a double-gridded computational domain $[15,16]$ :

$$
\phi_{c}\left(x_{i}, y_{j}\right)=h_{x} h_{y} \sum_{i=1}^{2 N_{x}} \sum_{j=1}^{2 N_{y}} G_{c}\left(x_{i}-x_{i^{\prime}}, y_{i}-y_{j^{\prime}}\right) \rho_{c}\left(x_{i^{\prime}}, y_{j^{\prime}}\right),
$$

where $i=1, \ldots, 2 N_{x}, j=1, \ldots, 2 N_{y}$, and

$\rho_{c}\left(x_{i}, y_{j}\right)= \begin{cases}\rho\left(x_{i}, y_{j}\right): & 1 \leq i \leq N_{x}, 1 \leq j \leq N_{y}, \\ 0: & N_{x}<i \leq 2 N_{x} \text { or } N_{y}<j \leq 2 N_{y},\end{cases}$ 


$$
\begin{aligned}
& G_{c}\left(x_{i}, y_{j}\right)=\left\{\begin{array}{l}
G\left(x_{i}, y_{j}\right): \\
G\left(x_{2 N_{x}-i+2}, y_{j}\right): \\
G\left(x_{i}, y_{2 N_{y}-j+2}\right): \\
G\left(x_{2 N_{x}-i+2}, y_{2 N_{y}-j+2}\right.
\end{array}\right. \\
& \rho_{c}\left(x_{i}, y_{j}\right)=\rho_{c}\left[x_{i}+2\left(L_{x}+h_{x}\right), y_{j}+2\left(L_{y}+h_{y}\right)\right], \\
& G_{c}\left(x_{i}, y_{j}\right)=G_{c}\left[x_{i}+2\left(L_{x}+h_{x}\right), y_{j}+2\left(L_{y}+h_{y}\right)\right] .
\end{aligned}
$$

These equations make use of the symmetry of the Green function in Eq. (18). From the above definition, one can show that the cyclic convolution will give the same electric potential as the convolution Eq. (19) within the original domain, i.e.,

$$
\phi\left(x_{i}, y_{j}\right)=\phi_{c}\left(x_{i}, y_{j}\right), \quad \text { for } i=1, N_{x} ; j=1, N_{y} .
$$

The potential outside the original domain is incorrect but is irrelevant to the physical domain. Since both $G_{c}$ and $\rho_{c}$ are periodic functions, the convolution for $\phi_{c}$ in Eq. (20) can be computed efficiently using an fast Fourier transform (FFT) as described by Hockney et al. [15].

In the above FFT-based algorithm, the particle domain and the electric field domain are contained in the same computational domain. Here, the particle domain is the configuration space containing the charged particles, and the field domain is the space where the electric field is generated by the charged particles in the particle domain. In the beam-beam interaction, two opposite-moving beams might not overlap with each other. For example, in the long-range interaction, the two colliding beams could be separated more than several $\sigma$ 's, where $\sigma$ is the rms size of the beam. The field domain where the electric field is generated by one beam can be different from the particle domain containing the beam. Figure 1 shows a schematic plot of the two separated domains.

In this figure, the particle domain has a range from $-R_{0}$ to $R_{0}$ for $x$ and $y$, and the field domain has a range
$1 \leq i \leq N_{x}+1,1 \leq j \leq N_{y}+1$,

$N_{x}+1<i \leq 2 N_{x}, 1 \leq j \leq N_{y}+1$,

$1 \leq i \leq N_{x}+1, N_{y}+1<j \leq 2 N_{y}$,

$N_{x}+1<i \leq 2 N_{x}, N_{y}+1<j \leq 2 N_{y}$, from 0 to $2 R_{0}$ for $x$ and $y$, where $R_{0}$ is the maximum extent of the beam. The origin of the field domain in this figure is $x_{c}=R_{0}, y_{c}=R_{0}$, where the origin is chosen to be at the beam centroid. In the beam-beam simulation, the origin of the field domain can be at an arbitrary location and varies from turn to turn. To apply Hockney's algorithm directly will require the computational domain to contain both the particle domain and the field domain, i.e., both beams. Since there is a large empty space between two beams, containing both beams in one computational domain will result in a poor spatial resolution of the beams. This is also computationally inefficient because the electric fields in the empty space between two beams are not used. To avoid this problem, we have defined a shifted Green function as

$$
G_{s}(x, \bar{x}, y, \bar{y})=-\frac{1}{2} \ln \left[\left(x_{c}+x-\bar{x}\right)^{2}+\left(y_{c}+y-\bar{y}\right)^{2}\right],
$$

where $x_{c}$ and $y_{c}$ are the center coordinates of the field domain. The electric potential in the field domain is written as

$$
\phi\left(x+x_{c}, y+y_{c}\right)=\int G_{s}(x, \bar{x}, y, \bar{y}) \rho(\bar{x}, \bar{y}) d \bar{x} d \bar{y} .
$$

Using the shifted Green function, the center of the field domain is shifted to the center of the particle domain. The ranges of $x$ and $y$ cover both the particle domain and the field domain in one computational domain. The FFT can be used to calculate the cyclic convolution in Eq. (20) using the new Green function. Here, on the doubled grids, the Green function is given as

$$
G_{c}\left(x_{i}, y_{j}\right)=-\frac{1}{2} \begin{cases}\ln \left[\left(x_{c}+x_{i}\right)^{2}+\left(y_{c}+y_{j}\right)^{2}\right]: & 1 \leq i \leq N_{x}, 1 \leq j \leq N_{y}, \\ \ln \left[\left(x_{c}-x_{2 N_{x}-i+2}\right)^{2}+\left(y_{c}+y_{j}\right)^{2}\right]: & N_{x}<i \leq 2 N_{x}, 1 \leq j \leq N_{y}, \\ \ln \left[\left(x_{c}+x_{i}\right)^{2}+\left(y_{c}-y_{2 N_{y}-j+2}\right)^{2}\right]: & 1 \leq i \leq N_{x}, N_{y}<i \leq 2 N_{y}, \\ \ln \left[\left(x_{c}-x_{2 N_{x}-i+2}\right)^{2}+\left(y_{c}-y_{2 N_{y}-j+2}\right)^{2}\right]: & N_{x}<i \leq 2 N_{x}, N_{y}<i \leq 2 N_{y} .\end{cases}
$$

Using the shifted Green function avoids the requirement that the particle domain and the field domain be contained in one big computational domain. This leads to better numerical resolution for the charge densities and the resulting electric fields than the conventional method, because the empty space between the beams is not included in the calculation. This is also far more efficient, in terms of computational effort and storage, than the traditional approach of gridding the entire problem domain.

As an example of the above FFT-based algorithms, we computed the radial electric field distribution generated by a round beam with a Gaussian density distribution using the particle domain and the field domain shown in Fig. 1. Figure 2 shows the radial electric field $E_{r}$ as a function of distance along the diagonal line of the particle domain using the conventional algorithm, i.e., without the shifted Green function, and $128 \times 128$ grid. The electric field from the analytical calculation is also given in the same figure for comparison. It is seen that the agreement between the numerical solution and the analytical calculation is excellent. As a test of the shifted 


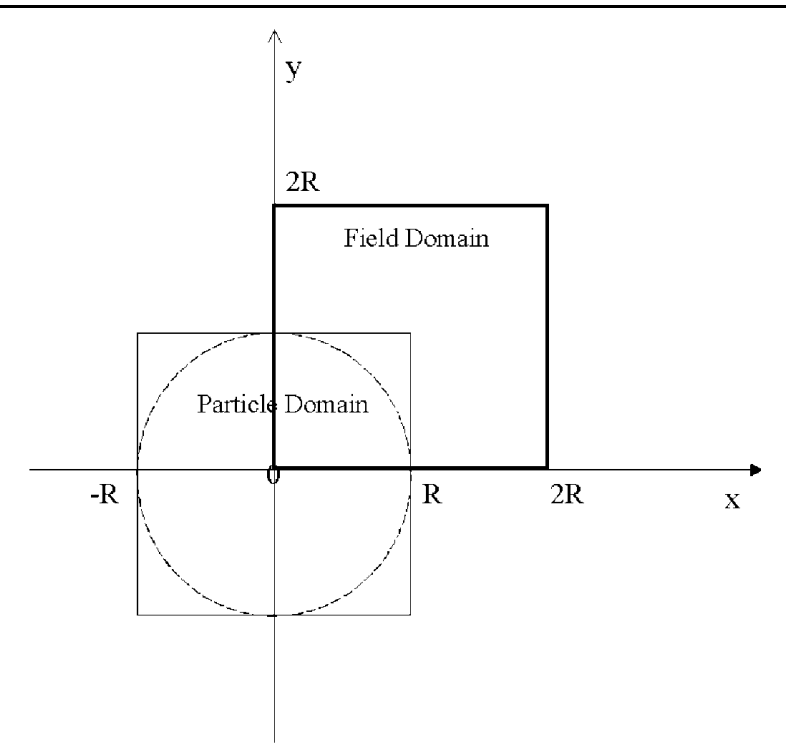

FIG. 1. A schematic plot of the particle domain and the field domain.

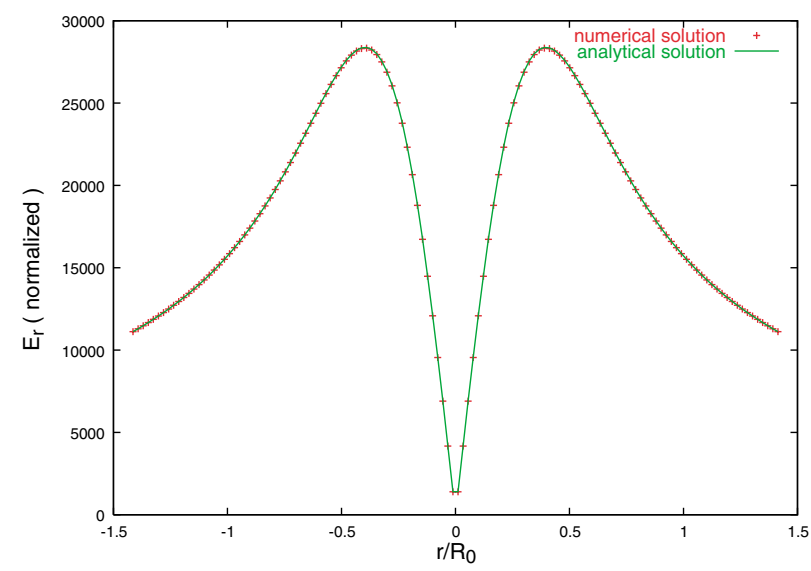

FIG. 2. (Color) Radial electric field as a function of distance along the diagonal line of the particle domain.

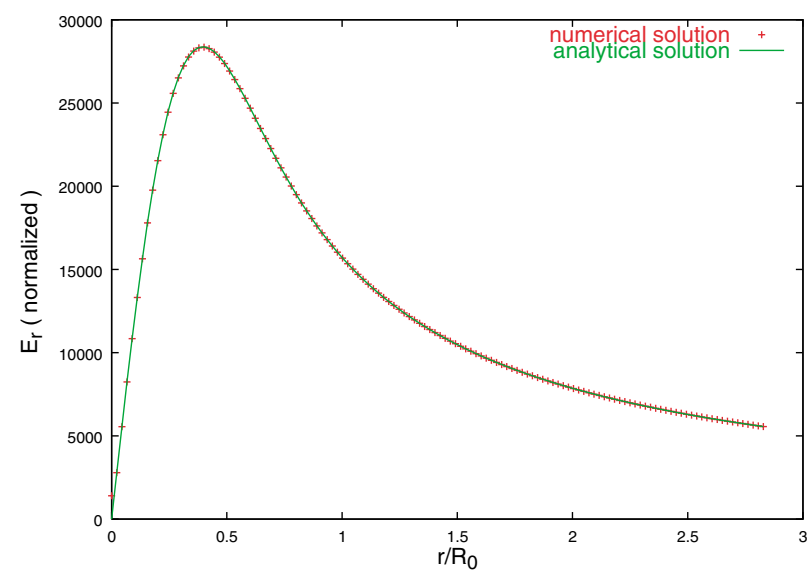

FIG. 3. (Color) Radial electric field as a function of distance along the diagonal line of the field domain.
Green function approach, we also computed the electric field $E_{r}$ along the diagonal line of the field domain. The results are shown in Fig. 3 together with the analytical calculation. We see that the two solutions are in excellent agreement.

\section{APPLICATIONS}

As a first application, we have studied the coherent beam-beam dipole oscillation in the proposed LHC. The nominal beam-beam parameters of the LHC are given in Table I.

In this study, the two proton beams are assumed to have the same physical parameters. The nominal beambeam parameter $\xi$ is -0.0034 . Here $\xi$ is defined as $-\left(r_{p} N \beta_{x}\right) /\left(4 \pi \gamma \sigma_{x}^{2}\right)$ and $r_{p}$ is the classical proton radius. Figure 4 shows the spectra of the sum and the difference of the beam centroids for the nominal LHC parameters. The normalized tune is defined as $\left(\nu-\nu_{x 0}\right) /|\xi|$, where $\nu_{x 0}$ is the horizontal bare tune, and where $\nu$ is the power spectrum associated with the sum or the difference of the centroid motion of the two beams. The simulation was run for 20000 turns using a single slice model for each beam with $1 \times 10^{6}$ particles and a $128 \times 128$ grid. The centroid of one beam is initially displaced by $0.01 \sigma$ on the horizontal plane and the vertical plane. Only head-on collisions were taken into account in this simulation. It is seen that there exist two oscillation frequencies for the centroid motion, one corresponding to the bare tune without beam-beam interaction (the $\sigma$ mode) and the other (the $\pi$ mode) with its frequency shifted downwards in tune by $(1.19 \pm 0.015)|\xi|$ in $x$ and by $(1.19 \pm 0.015)|\xi|$ in $y$, where the uncertainty 0.015 is due to the finite number of turns used in the simulation. The frequency downshift has also been calculated using a linearized Vlasov analysis which gives $1.21 \xi$ [17]. We see that the simulation result agrees with the theoretical calculation within $2 \%$.

The same model was also employed to simulate the long-range parasitic collisions in the LHC. To reduce the computing time, we lumped all parasitic collisions into a single collision [8]. A static dipole kick from the longrange collision was subtracted following the treatment of Herr et al. [14]. The beam-beam kick in the long-range

TABLE I. LHC nominal beam-beam parameters.

\begin{tabular}{lc}
\hline \hline Beam energy $(\mathrm{TeV})$ & 7 \\
Protons per bunch & $1.05 \times 10^{11}$ \\
$\beta^{*}(\mathrm{~m})$ & 0.5 \\
rms spot size at the IP $(\mu \mathrm{m})$ & 15.9 \\
Betatron tunes $\left(\nu_{x}, \nu_{y}\right)$ & $(0.31,0.32)$ \\
rms bunch length $(\mathrm{m})$ & 0.077 \\
Synchrotron tune $\nu_{z}$ & 0.0021 \\
\hline \hline
\end{tabular}



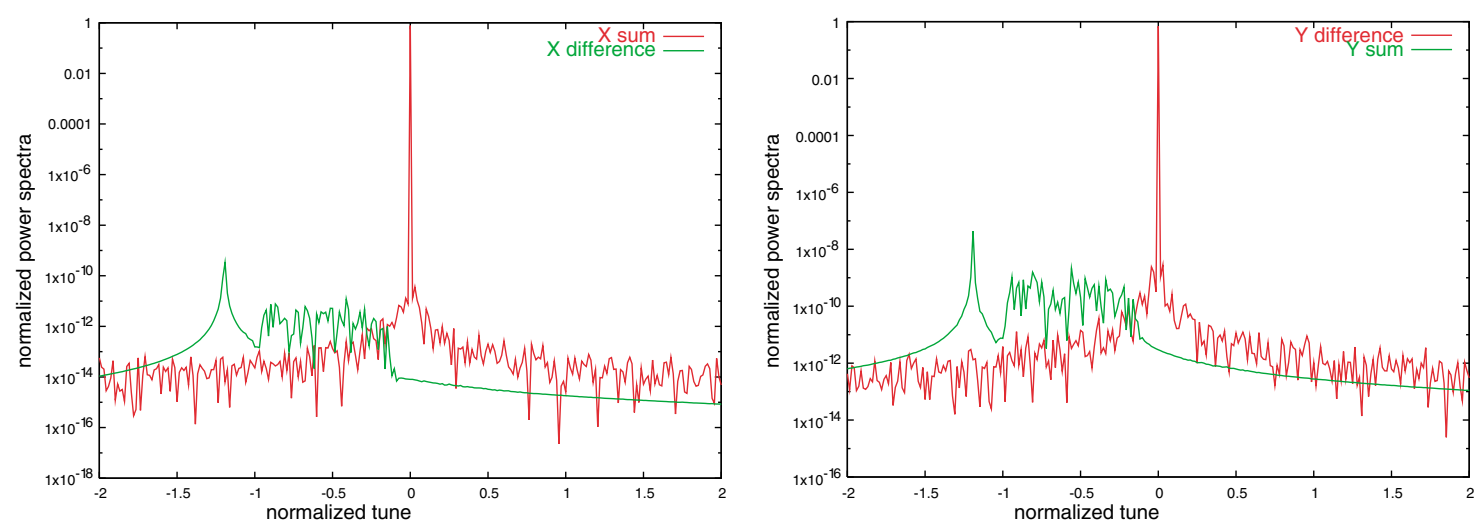

FIG. 4. (Color) Spectra of the horizontal (left panel) and vertical (right panel) sum and the difference centroid motion for nominal beam-beam collision parameters.
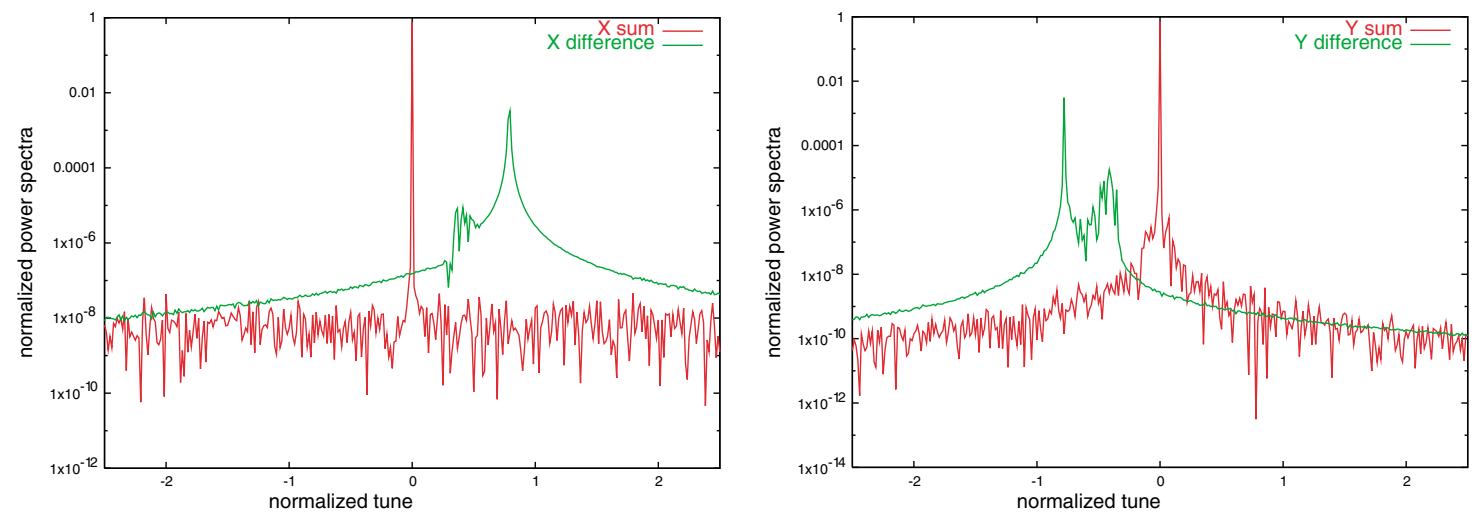

FIG. 5. (Color) Spectra of the horizontal (left panel) and vertical (right panel) sum and difference centroid motion for long-range beam-beam collision with $9 \sigma_{x}$ horizontal separation.

interaction is given by

$$
\begin{aligned}
& \Delta x_{2,1}^{\prime}=n_{\mathrm{par}} \frac{2 q_{1} q_{2} N_{1,2}}{\gamma_{2,1} 4 \pi \epsilon_{0} m_{2,1} c^{2}}\left\{E_{x_{1,2}}-D \frac{1}{L_{x} \sigma_{x}}\left[1.0-\exp \left(-\frac{L_{x}^{2}}{2}\right)\right]\right\}, \\
& \Delta y_{2,1}^{\prime}=n_{\mathrm{par}} \frac{2 q_{1} q_{2} N_{1,2}}{\gamma_{2,1} 4 \pi \epsilon_{0} m_{2,1} c^{2}}\left\{E_{y_{1,2}}-D \frac{1}{L_{y} \sigma_{y}}\left[1.0-\exp \left(-\frac{L_{y}^{2}}{2}\right)\right]\right\},
\end{aligned}
$$

where $L_{x} \sigma_{x}$ is the horizontal separation distance of the two beams, $L_{y} \sigma_{y}$ is the vertical separation distance, $D$ is a sign function depending on the relative position of the two beams, and $n_{\mathrm{par}}$ is the number of parasitic collisions. In the above equations, the static dipole kick is approximated by a constant dipole kick generated by a Gaussian distribution at a distance $x=L_{x} \sigma_{x}$ or $y=$ $L_{y} \sigma_{y}$. Figure 5 shows the spectra of the sum and the difference of the beam centroids with $9 \sigma_{x}$ horizontal separation. Here, only long-range interactions are included with 16 parasitic collisions. In the horizontal plane, the $\pi$ mode frequency is shifted upward instead of downward as opposed to the no-separation collision case in Fig. 4. The normalized tune shifts of the $\pi$ mode are $(0.79 \pm 0.015,-0.78 \pm 0.015)$. The numerical parameters are the same as those used in the nominal LHC simulation without separation. Since the transverse distance between the two beams at the parasitic collision points is significantly larger than the rms size of the beams, the effects of the beam distribution on the coherent motion might not be important. The beams might be treated as rigid bunches. In this case, the contribution of the long-range beam-beam interaction to the tune shift of the coherent oscillation modes would be twice the incoherent long-range tune shift, which is proportion to $1 / L_{x}^{2}$. Figure 6 shows the values of the $\pi$ mode tune shifts at several different horizontal separations, along with a curve having the $1 / L_{x}^{2}$ dependence. We see that the tune shifts fit the $1 / L_{x}^{2}$ scale very well. 


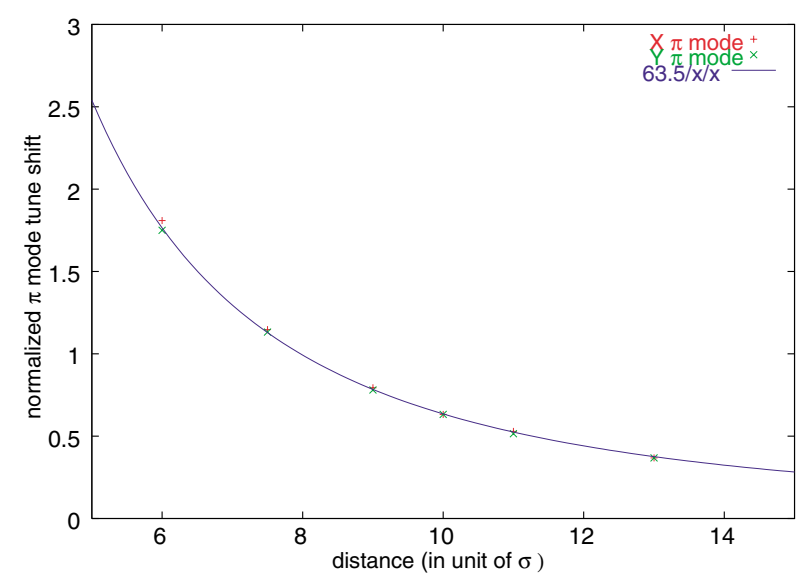

FIG. 6. (Color) Normalized $\pi$ mode tune shift as a function of horizontal separation for the case of long-range beam-beam collisions.

In the luminosity monitoring scheme being developed at LBNL for the LHC, one beam is deliberately swept in a circle about the geometrical collision point, where the other beam remains fixed $[18,19]$. This sweeping can be achieved by an appropriate time-dependent closed orbit bump spanning the interaction point. As a test, we have chosen a sweeping period of 1000 turns and a sweeping radius of $0.6 \sigma_{0}$ for the closed orbit of beam 2 , while the closed orbit of beam 1 remains static and is offset by $0.2 \sigma_{0}$ from the nominal interaction point at $45^{\circ}$ relative to the horizontal axis. Figure 7 shows the luminosity per collision as a function of turns during the process of sweeping. It is seen that the luminosity oscillates due to the off-center collisions with a period of 1000 turns. In practice, this is the signal that could be used to optimize the luminosity. Figure 8 shows the normalized rms emittances as a function of turns during the sweeping process. There is no significant emittance growth after 90000 turns except for a slightly initial increase that can be attributed to particle redistribution. However, we have

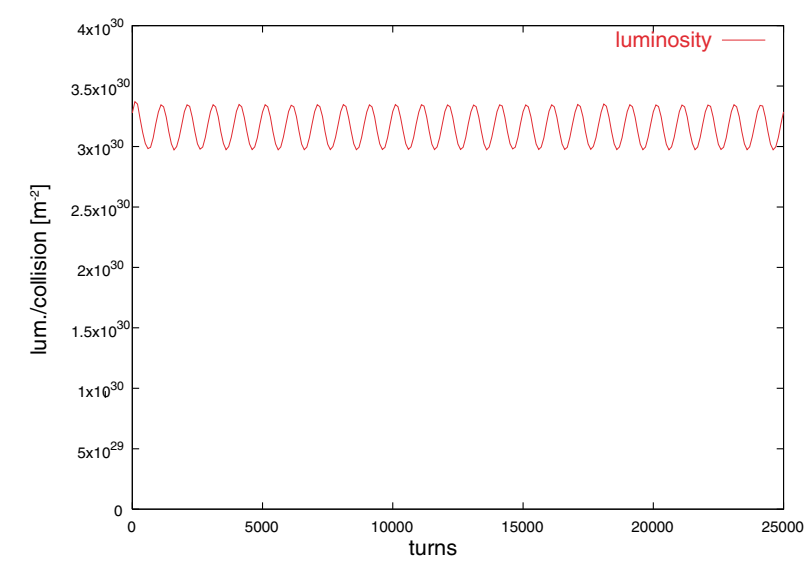

FIG. 7. (Color) The luminosity per collision as a function of turns during the sweeping process.

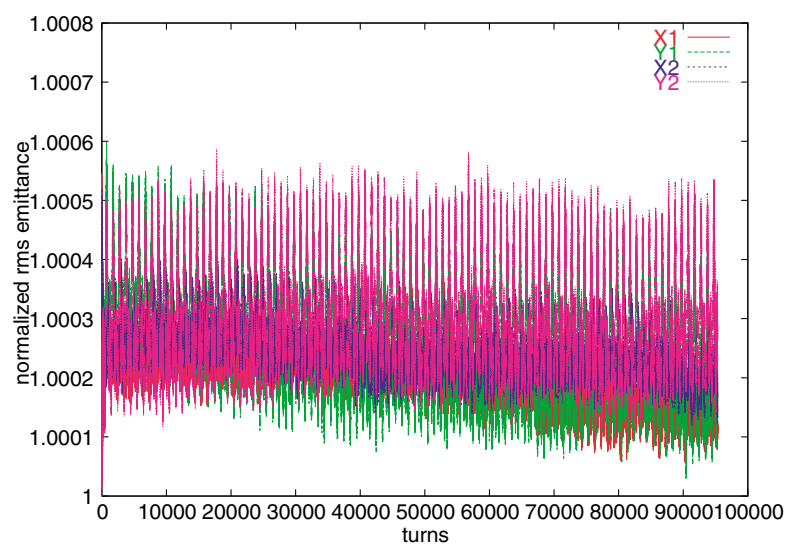

FIG. 8. (Color) The rms emittance as a function of turns during the sweeping process.

found that, when the number of charged particles per bunch is increased by a factor of $10,2 \%-4 \%$ emittance growth is observed after 90000 turns. Figure 9 shows the beam centroid spectra of two sweeping beams. Comparing with the nominal case in Fig. 4, we see that the tune shifts of the $\pi$ modes are smaller during the sweeping operation owing to the lower effective beambeam parameter. The difference spectra also show sidebands of the $\pi$ modes separated by 0.001 , corresponding to the sweeping tune.

\section{CONCLUSIONS}

In this paper, we have presented an FFT-based method to evaluate the electromagnetic field in the study of the strong-strong beam-beam interaction. Using such a method, we can handle not only the on-axis head-on beam-beam collision but also the long-range beambeam collision with arbitrary separation without losing computational efficiency. A study of the coherent tune shift for the beam-beam interaction shows good agreement with the theoretical prediction. As an application,

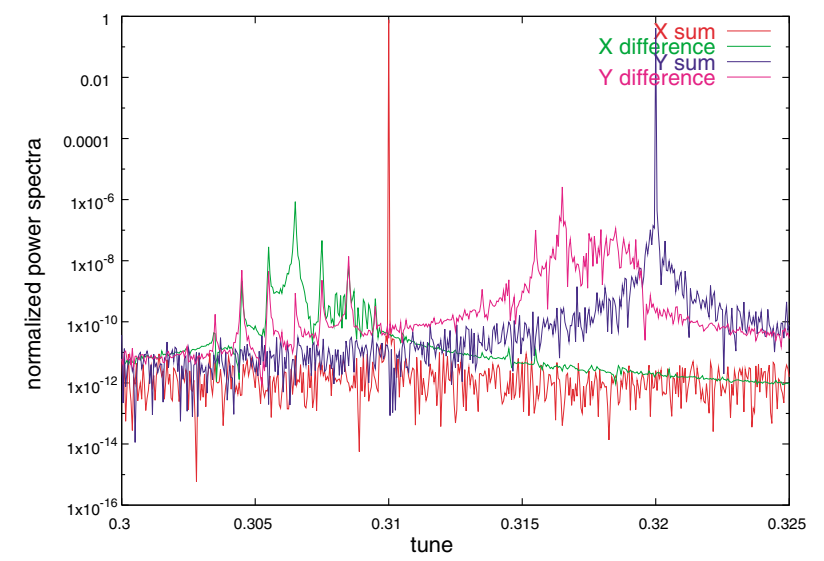

FIG. 9. (Color) Spectra of the sum and difference centroid motion during the sweeping process. 
we have also studied a luminosity monitoring scheme for LHC based on beam sweeping. In our simulations, there is no significant beam quality degradation after 90000 turns for the nominal parameter values.

\section{ACKNOWLEDGMENTS}

The authors would like to thank Dr. A. Adelmann for reading the manuscript. The research used resources of the National Energy Research Scientific Computing Center, which is supported by the Office of Science of the U.S. Department of Energy (U.S. DOE/SC) under Contract No. DE-AC03-76SF00098. This work was performed under the auspices of a Scientific Discovery through Advanced Computing project, "Advanced Computing for 21st Century Accelerator Science and Technology, which is supported by the U.S. DOE/SC Office of High Energy and Nuclear Physics and the Office of Advanced Scientific Computing Research.

[1] K. Hirata, H. Moshammer, and F. Ruggiero, Part. Accel. 40, 205 (1993).

[2] K. Hirata, Phys. Rev. Lett. 20, 2228 (1995).

[3] M. A. Furman, A. Zholents, T. Chen, and D. Shatilov, CBP Tech Note No. 59, 1996.

[4] Y. Papaphilippou and F. Zimmermann, Phys. Rev. ST Accel. Beams 2, 104001 (1999).
[5] M. A. Furman, LBNL Report No. LBNL-42669, CBP Note No. 272, 1999.

[6] T. Koyama, Phys. Rev. ST Accel. Beams 2, 024001 (1999).

[7] L. H. A. Leunissen, F. Schmidt, and G. Ripken, Phys. Rev. ST Accel. Beams 3, 124002 (2000).

[8] M. P. Zorzano and F. Zimmermann, Phys. Rev. ST Accel. Beams 3, 044401 (2000).

[9] S. Krishnagopal and R. Siemann, Phys. Rev. Lett. 28, 2461 (1991).

[10] E. B. Anderson, T. I. Banks, and J.T. Rogers, in Proceedings of the 1999 Particle Accelerator Conference, New York (IEEE, Piscataway, NJ, 1999), p. 1686.

[11] J. Shi and D. Yao, Phys. Rev. E 62, 1258 (2000).

[12] K. Ohmi, Phys. Rev. E 62, 7287 (2000).

[13] Y. Cai, A. W. Chao, S. I. Tzenov, and T. Tajima, Phys. Rev. ST Accel. Beams 4, 011001 (2001).

[14] W. Herr, M. P. Zorzano, and F. Jones, Phys. Rev. ST Accel. Beams 4, 054402 (2001).

[15] R.W. Hockney and J. E. Eastwood, Computer Simulation Using Particles (McGraw-Hill, New York, 1985).

[16] J.W. Eastwood and D. R. K. Brownrigg, J. Comput. Phys. 32, 24 (1979).

[17] K. Yokoya and H. Koiso, Part. Accel. 27, 181 (1990).

[18] S. Krishnagopal, M. A. Furman, and W. C. Turner, LBNL Report No. LBNL-43061, CBP Note No. 308, 1999.

[19] M. A. Furman and W.C. Turner, LBNL Report No. LBNL-46223, CBP Note No. 350, 2000. 